\title{
List of delegations
}

(C) European Association for Predictive, Preventive and Personalised Medicine 2011

- Organisation of United Nations, $\boldsymbol{U N}$, www.un.org

- World Health Organisation, WHO, www.who.int

- EU-Commission, http://ec.europa.eu

- The Innovative Medicines Initiative, IMI, http://eo.imi-europe.org

- Early Detection Research Network, EDRN NIH / NCI, http://edrn.nci.nih.gov

- European Society of Radiology, $\boldsymbol{E S R}$, www.myesr.org

- European Medical Association, $\boldsymbol{E} \boldsymbol{M} \boldsymbol{A}$, www.emanet.org

- "Brains for Brain" Research Foundation, B4B http://mldfoundation.org

- European Federation of Neurological Associations,

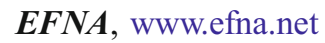

- European Brain Council, $\boldsymbol{E B C}$, www.europeanbraincouncil.org
- Niemann Pick C International Association, www.niemannpick.org.uk

- European Society of Predictive Medicine, EUSPM, http://www.euspm.org

- German Federation of Diabetics, DDB, http://www.diabetikerbund.de

- European Diagnostic Manufacturers Association, $\boldsymbol{E D} \boldsymbol{M} \boldsymbol{A}$, www.edma-ivd.be

- European Biopharmaceutical Enterprises, EBE-Biopharma, http://www.ebe-biopharma.org

- EuroBioForum, www.eurobioforum.eu

- Computer Assisted Radiology and Surgery,

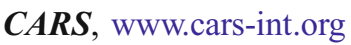

- European, Middle Eastern \& African Society for Biopreservation \& Biobanking, $\boldsymbol{E S B} \boldsymbol{B}$, http://www.esbb.org

- Alexander von Humboldt-Foundation, www.humboldt-foundation.de 